\title{
EDITORIAL
}

\section{Withdrawal of Saudi trainees exposes vulnerability of Canadian health care}

\author{
M. Hashim Khan MBBS, Nadine Abdullah MD MEd, Matthew B. Stanbrook MD PhD
}

Cite as: CMAJ 2018 September 4;190:E1030. doi: 10.1503/cmaj.181084

After early online release of this editorial on August 21, the Saudi government granted extensions to the affected residents and fellows to continue their medical training in Canada until further arrangements are made.

$\mathbf{0}$

n Aug. 3, 2018, a diplomatic row erupted between Saudi Arabia and Canada following a human rights-related tweet by Canada's minister of foreign affairs. Among several retaliatory measures, Saudi Arabia announced the discontinuation of scholarships to and imminent relocation of about 16000 Saudi students in Canada, including 800 medical trainees. The sudden, profound impact of this decision is being felt not only by Saudi trainees, but also by those who have come to rely on them: other trainees and physicians at academic medical training programs and health care institutions across Canada, and, ultimately, Canadian patients.

For decades, Canadian medical institutions have welcomed thousands of internationally funded residents and fellows, predominantly from Saudi Arabia and other Middle Eastern countries, in a mutually beneficial training relationship. The best and brightest among them succeed in competing for the privilege of training in our world-class academic institutions, provide years of clinical care and research productivity at no cost to Canadian taxpayers, then bring the advanced skills and professional values of Canadian medicine back to their home countries. This partnership has led to ongoing professional collaborations, longstanding friendships and greater understanding of one another's cultures. Some trainees have even gone on to make Canada their new home, a testament to both the quality of their educational experiences and how they have been welcomed by Canadians.

Because of their government's action, Saudi trainees who worked hard to earn this invaluable opportunity have had their whole lives uprooted abruptly. Besides having to relocate entire families with mere weeks' notice and imminent loss of funding, they face disrupted training and an uncertain future. Many, despite completing years of study and clinical training, will be unable to write Canadian subspecialty examinations this fall. A few will not be able to continue practising medicine. Ultimately, Saudi citizens who were to benefit from being cared for by Canadian-trained physicians stand to lose as well.

As the medical community has responded overwhelmingly with shock, sympathy and support for our Saudi colleagues, academic health centres have been scrambling to adjust service and on-call coverage left vacant. Saudis currently account for $5 \%-15 \%$ of postgraduate medical trainees across institutions, but the proportion is much higher within some specialty programs. The impact this will have on safe and timely health care delivery to Canadian patients remains to be seen, but an effect on physicians and other trainees in these centres in terms of workload and stress is already being felt. The academic mission of the affected institutions to deliver education, research, innovation and advanced care will inevitably be strained as well.

Earlier this year, a record number of Canadian medical graduates went unmatched to many of the same residency programs now losing their Saudi trainees. Being internationally funded, the now-vacant spots cannot now be filled by Canadians - this would require Canadian funding. Internationally funded trainees have never competed with Canadians in the match for residency training spots. However, as a substantial (and, until now, reliable) source of funding, they have likely been a factor enabling our governments to erode the number of funded residency positions. What began as a symbiotic relationship may have led to a dangerous dependency, evolving now to the paradox of simultaneously understaffed programs and unmatched trainees.

Canada's health care and education systems must never be vulnerable to the spontaneous decisions of a foreign government. This event must serve as a wake-up call to federal and provincial governments, which should act rapidly to increase the number of funded residency and fellowship positions for Canadian medical students and Canadian graduates of foreign medical schools. Our ability to meet the present and future health care needs of our population, while safeguarding the mission of our academic centres, is not something we can afford to contract out.

The loss of our internationally funded trainees highlights more clearly than ever how valuable they have been. Our profession and our country must express gratitude for the service and contributions of these trainees over the years to Canadian medicine and the Canadian people. Canada will continue to welcome internationally funded trainees - including Saudis, as future diplomatic relations allow but should do so because we want them, not because we need them.

Competing interests: See www.cmaj.ca/site/misc/cmaj_staff.xhtml for Matthew Stanbrook. Nadine Abdullah is a site director for the Internal Medicine Residency Program at the University of Toronto. M. Hashim Khan reports that he is a graduate of the internal medicine and respirology residency training programs at the University of Toronto and is presently a clinical fellow at the University of Toronto.

Affiliations: Department of Medicine (Khan, Abdullah, Stanbrook), University of Toronto, Toronto, Ont.; Deputy editor (Stanbrook), CMAJ; ICES (Stanbrook), Toronto, Ont.

Correspondence to: CMAJ editor, cmaj@cmajgroup.ca 